\title{
Knowledge and Attitude toward Orthodontic Treatment among treated Subjects compared with Untreated Subjects and Correlation with Patient-Orthodontist Communication
}

\author{
${ }^{1} \mathrm{M}$ Essamet, ${ }^{2}$ Ismail A Darout
}

\begin{abstract}
Aim: This study was designed to evaluate knowledge and awareness toward orthodontic treatment among treated subjects as compared with untreated subjects and to correlate the communications between treated subjects and their practicing orthodontists.
\end{abstract}

Materials and methods: Self-reported questionnaires were distributed to be completed by the consent subjects. They were 340 untreated subjects (220 males and 120 females) and 102 subjects ( 57 males and 45 females) who recently received orthodontic treatment in the age range 19 to 28 years. Subjects that received orthodontic treatment were selected among the attendees of clinics of College of Dentistry, Jazan University, Kingdom of Saudi Arabia. The data were analyzed using Statistical Package for the Social Sciences (version 21, Institute Inc., USA).

Results: Subjects who received orthodontic treatment scored higher mean of total scores of awareness 14.74 and knowledge 11.18. The difference of awareness and knowledge between treated and untreated subjects was highly significant $(p=0.000)$. The female treated subjects scored higher than male treated subjects in attitude toward thumb sucking as a cause of malocclusion ( 91.3 and $70.2 \%$ respectively). Early primary teeth extraction as a cause of malocclusion was scored by $76.1 \%$ females and $42.1 \%$ males. The male treated subjects show significant $(p>0.05)$ correlation between the total items of knowledge of orthodontic treatment and total items of orthodontist-patient communications.

Conclusion: Orthodontic treated subjects have more knowledge and awareness about orthodontic treatment than untreated subjects. A significant association was found between communications and knowledge of orthodontic treatment in male as compared with female patients.

Clinical significance: Knowledge and awareness toward orthodontic treatment and the communications of orthodontic patients with treating orthodontist can significantly increase the orthodontic treatment outcome.

${ }^{1}$ Division of Orthodontics, Department of Preventive Dental Sciences, College of Dentistry, Jazan University, Jazan, Kingdom of Saudi Arabia

${ }^{2}$ Division of Periodontics, Department of Preventive Dental Sciences, College of Dentistry, Jazan University, Jazan, Kingdom of Saudi Arabia

Corresponding Author: Ismail A Darout, Division of Periodontics, Department of Preventive Dental Sciences College of Dentistry, Jazan University, Jazan, Kingdom of Saudi Arabia, e-mail: idarout1959@gmail.com
Keywords: Awareness, Behavior, Esthetic problem, Malocclusions, Oral health.

How to cite this article: Essamet M, Darout IA. Knowledge and Attitude toward Orthodontic Treatment among treated Subjects compared with Untreated Subjects and Correlation with Patient-Orthodontist Communication. World J Dent 2017;8(3):218-223.

\section{Source of support: Nil}

Conflict of interest: None

\section{INTRODUCTION}

In a modern world teeming with social media websites and interactive communications, there is significantly increased awareness and attention to facial appearance and smile beauty. Thus, the need to make orthodontic correction among young population has become prevalent. In addition to its impact on the psychosocial wellbeing, a positive facial appearance and attractive smile increase the confidence and self-esteem. ${ }^{1}$ The orthodontic treatment objective is to correct the malocclusion which might affect the facial and dental esthetics. However, the success of the process might need a positive behavior and cooperation of the patient and treating orthodontist. In addition, the awareness, attitude, and knowledge of orthodontic patients about oral health care are a perquisite for

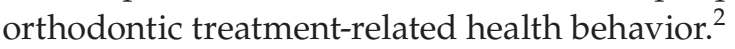

Orthodontic treated patients in the Netherlands were found to have a significantly more positive attitude and knowledge toward orthodontic treatment than untreated subjects. ${ }^{3}$ Understanding the behaviors and attitudes of the patient can provide valuable information for the orthodontist prior to developing treatment plan and orthodontic treatment. The comparison between previously treated subjects and untreated subjects helps to find the determinants of the awareness and attitude of orthodontic patients toward the orthodontic treatment. Previously, Essamet and Darout ${ }^{4}$ indicated an acceptable perception of oral health care behavior related to orthodontic treatment among untreated students of Jazan University in Saudi Arabia.

Orthodontic treatment may require long duration, and the compliance of orthodontic patients for the successful 
treatment outcome is mostly needed. However, many orthodontists have difficulty to deal with behavioral compliance of their patients. Thus, orthodontists seem to deal with the complex issue of motivating and improving the compliance of their patients during the appointments. These involve educating the patients and/or parents about the importance of compliance. ${ }^{4,5}$ Moreover, the orthodontists need to verbally praise for compliant behavior. ${ }^{6,7}$ Treating the patients with respect, establishing a good communication, ${ }^{8,9}$ and informing the patients about the progress of treatment offered to them are rewarded as well. ${ }^{10,11}$ However, orthodontic patients differ psychologically from each other in the perception of the way their practicing orthodontist contacts and communicates with them.

In the Kingdom of Saudi Arabia, communication with female patients represents a serious challenge for male orthodontists because of local culture and traditions. Moreover, the English languish might be a barrier for nonArabic-speaking orthodontists, making patient-orthodontist communication quite insufficient. Little information is available online that evaluated the communications of Saudi orthodontic treated patients with their treating orthodontists. Hence, this study was designed to evaluate the communications of orthodontic patients and their treating orthodontist, and test its correlation with knowledge and awareness toward the orthodontic treatment.

The aims of this study were to evaluate the knowledge, awareness, and attitude toward orthodontic treatment in previously treated subjects and to compare them with untreated subjects and to evaluate the communication between previously treated subjects and their practicing orthodontists and correlate them with the knowledge and awareness.

\section{MATERIALS AND METHODS}

\section{Study Subjects}

The subjects involved untreated and recently received orthodontic treatment. The untreated subjects were students of medical and health sciences colleges and nonmedical colleges of Jazan University. They were 340 (220 males and 120 females) subjects as described elsewhere by Essamet and Darout. ${ }^{4}$ Those who recently received orthodontic treatment were selected among the attendees of clinics of College of Dentistry, Jazan University. They were 102 (57 males and 45 females) subjects. In total, 442 young subjects (277 males and 165 females) with similar demographic aspects participated in this questionnaire-based study. The knowledge and attitude toward orthodontic treatment as well as patient-orthodontist communication between untreated and treated subjects were compared. In the previous study by Essamet and Darout, ${ }^{4}$ students from Jazan
University were used to evaluate the knowledge and awareness toward the oral health behavior. Therefore, it was decided to use the previous subjects, called untreated subjects, and recently received orthodontic treatment subjects to compare items of knowledge and attitude toward orthodontic treatment as well as patient-orthodontist communications. Subjects were having similar demographic characteristics.

\section{Questionnaire}

A questionnaire consisting of 16 items was distributed to all the participated subjects to be completed by consent subjects. Eight questions were used to examine the awareness of both groups toward the orthodontic treatment, and same number of questions was used for the knowledge of orthodontic treatment. For the group of previously treated subjects, the reliability of the questionnaire and the validity of its Arabic version were verified on 20 subjects, whereas the reliability and validity of the questionnaire for untreated subjects were examined in a previous study. The response of the subjects to the questions was recorded on a 3-point Likert scale (a. Yes, b. No, and c. do not know). The second questionnaire of seven items was distributed only to the previously treated subjects and was used to evaluate the patient-orthodontist communication.

\section{Statistical Analysis}

The data were collected and entered into Excel sheet. Using Statistical Package for the Social Sciences version 21 (Illinois, Chicago, USA), the items and characteristics of the questionnaire were determined. A chi-square test was used to examine the differences in item level. Independent sample Student's t-test was used to determine the difference in sum scores for previously treated subjects and untreated subjects over all items of awareness and knowledge $(\mathrm{p}=0.05)$. Bivariate correlation was used to test the correlation between the patient-orthodontist communication of previously treated subjects and their awareness and knowledge of orthodontic treatment.

\section{RESULTS}

The previously treated subjects in Table 1 recorded higher mean of total score of awareness (14.74) and knowledge

Table 1: Mean and SD of untreated subjects $(n=340)$ and previously treated subjects $(n=102)$ and Student's t-test for differences of total items of awareness and knowledge between the two groups

\begin{tabular}{lllll}
\hline & \multicolumn{2}{c}{ Untreated subject } & & \multicolumn{2}{c}{ Treated subject } \\
\cline { 2 - 2 } \cline { 5 - 6 } & Mean $\pm S D$ & & Mean $\pm S D$ & p-value \\
\hline Awareness & $10.51 \pm 3.586$ & & $14.74^{*} \pm 1.960$ & 0 \\
Knowledge & $8.55 \pm 3.017$ & & $11.18^{*} \pm 2.649$ & 0 \\
\hline
\end{tabular}

*Difference was statistically significant $(p<0.05)$; SD: Standard deviation 
Table 2: Frequency and percentage of positive awareness and knowledge of orthodontic treatment between untreated subjects and previously treated subjects

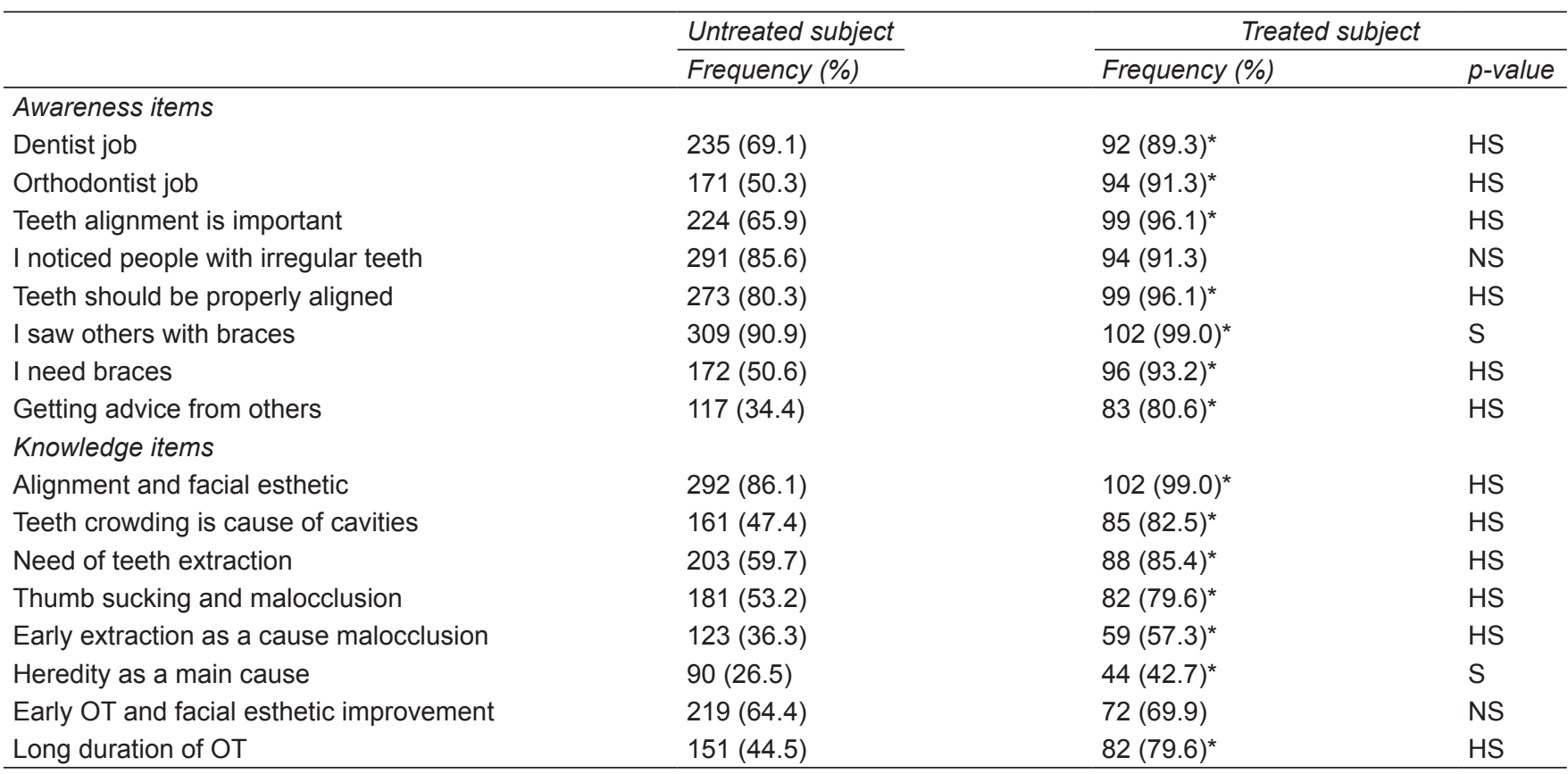

*Variable group scored more positive awareness and knowledge of orthodontic treatment; P: Pearson Chi-square; S: Significant; HS: Highly significant; NS: Not significant

(11.18). The Student's t-test shows highly significant differences for the total score of awareness and knowledge of orthodontic treatment, and the difference of awareness and knowledge was very significant between previously treated subjects and untreated subjects $(\mathrm{p}=0.000)$.

For the 16 items, Table 2 shows the frequency and the percentage of positive awareness and knowledge of orthodontic treatment for untreated and treated subjects. The treated subjects scored higher in all items except their perception of teeth crowding and information regarding the importance of early orthodontic treatment, wherein the differences were less significant. Although the differences in all items of awareness and knowledge were significant between untreated and treated subjects, the perception people with irregular teeth and the attitude toward early orthodontic treatment and facial esthetic improvement were not significant at all.

Gender differences were not found in group of previously treated subjects for all eight items of awareness and six items of knowledge, as shown in Table 3. However, the female treated subjects scored higher than male treated subjects in attitude toward thumb sucking and malocclusion $(91.3$ and $70.2 \%$ for females and males respectively, with $\mathrm{p}=0.008$ ) and early primary teeth extraction as a cause of malocclusion (76.1 and $42.1 \%$ for females and males respectively, with $\mathrm{p}=0.001$ ).

Table 4 shows high scores for the communication between the previously treated subjects and their respective treating orthodontist. However, gender differences of orthodontist-patient communication were not found.
The male treated subjects recorded highly significant correlation between the total items of knowledge of orthodontic treatment and total items of orthodontistpatient communication $(\mathrm{p}>0.05)$, as shown in Table 5 . However, no significant correlations between the male and female orthodontic patients regarding the total items of awareness and patient-orthodontist communication were found.

\section{Ethical Considerations}

Ethical approval was obtained from the College of Dentistry Research Office. A written informed consent was obtained from the participants. The participants were told that there is no direct benefit of their participation; however, knowledge gained may lead to the prevention and treatment of malocclusion and that their information for this research will be confidential and will not be disclosed to others.

\section{DISCUSSION}

Orthodontic treatment is an elective treatment for malocclusion and the need for positive perception and attitude toward orthodontic treatment is highly demanding from the patient at the commencement. A successful outcome of orthodontic treatment may be a result of good compliance from the patients to follow the recommended regimens suggested by the orthodontist. ${ }^{11}$ The patients must take care of their oral hygiene and follow the dietary restrictions and care about their removable appliances during 
Knowledge and Attitude toward Orthodontic Treatment among treated Subjects compared with Untreated Subjects

Table 3: Numbers $(n)$ and percentage (\%) of positive awareness and knowledge of orthodontic treatment between male (57) and female (45) previously treated subjects

\begin{tabular}{|c|c|c|c|}
\hline \multirow[b]{2}{*}{ Items } & \multirow{2}{*}{$\frac{\text { Male }(n=57)}{n(\%)}$} & \multicolumn{2}{|c|}{ Female $(n=45)$} \\
\hline & & $n(\%)$ & $p$-value \\
\hline \multicolumn{4}{|l|}{ Awareness items } \\
\hline Dentist job & $49(86.0)$ & $43(93.5)$ & NS \\
\hline Orthodontist job & $50(87.7)$ & $44(95.7)$ & NS \\
\hline Teeth alignment is important & $53(93.0)$ & $46(100.0)$ & NS \\
\hline I noticed people with irregular teeth & $50(87.7)$ & $44(95.7)$ & NS \\
\hline Teeth should be properly aligned & $55(96.5)$ & $44(95.7)$ & NS \\
\hline I saw others with braces & $57(100.0)$ & $45(97.8)$ & NS \\
\hline I need braces & $52(91.2)$ & $44(95.7)$ & NS \\
\hline Getting advice from others & $44(77.2)$ & $39(84.8)$ & NS \\
\hline \multicolumn{4}{|l|}{ Knowledge items } \\
\hline Alignment and facial esthetic & $56(98.2)$ & $46(100.0)$ & NS \\
\hline Teeth crowding is cause of cavities & $43(75.4)$ & $42(91.3)$ & NS \\
\hline Need of teeth extraction & $47(82.5)$ & $41(89.1)$ & NS \\
\hline Thumb sucking and malocclusion & $40(70.2)$ & $40(91.3)^{*}$ & $S$ \\
\hline Early extraction as a cause malocclusion & $24(42.1)$ & $35(76.1)^{*}$ & HS \\
\hline Heredity as a main cause & $21(36.8)$ & $23(50.0)$ & NS \\
\hline Early OT and facial esthetic improvement & $39(68.4)$ & $33(71.7)$ & NS \\
\hline Long duration of OT & $45(78.9)$ & $37(80.4)$ & NS \\
\hline
\end{tabular}

S: Significant; HS: Highly significant; NS: Not significant; P: Pearson Chi-square

Table 4: Frequency and percentage of positive communication between orthodontist and patient between male (57) and female (45) previously treated subjects

\begin{tabular}{lllll}
\hline & \multicolumn{2}{l}{ Males } & & \multicolumn{2}{c}{ Females } \\
\cline { 2 - 3 } Communication items & Frequency (\%) & Frequency (\%) & p-value \\
\hline 1 & $57(100)$ & $43(95.6)$ & NS \\
2 & $52(91.2)$ & $42(93.3)$ & NS \\
3 & $48(84.2)$ & $38(86.4)$ & NS \\
4 & $41(71.9)$ & $34(75.6)$ & NS \\
5 & $50(89.3)$ & $43(95.6)$ & NS \\
6 & $54(94.7)$ & $41(91.1)$ & NS \\
7 & $49(86)$ & $36(81.8)$ & NS \\
\hline
\end{tabular}

NS: Not significant; P: Pearson Chi-square

orthodontic treatment. ${ }^{12,13}$ In this study, the knowledge and awareness toward orthodontic treatment among untreated and treated subjects as well as the orthodontist and treated patients' communications were evaluated.

The results of this study show no significant differences between subjects who recently received orthodontic treatment and untreated ones in knowledge and awareness items toward orthodontic treatment. Previously,
Essamet and Darout ${ }^{4}$ demonstrated that subjects without orthodontic experience have acceptable attitude toward orthodontic treatment. Regarding the general attitude toward orthodontic treatment, this study shows statistically significant differences between previously treated subjects and untreated subjects. This finding is consistent with a previous study. ${ }^{3}$

Gender equality in general items of awareness and the knowledge of orthodontic treatment was demonstrated in our study. Thus, both males and females recently receiving orthodontic treatment reported equal positive attitude toward the orthodontic experience. However, the female subjects showed more knowledge on the impact of thumb sucking and the early extraction of primary teeth on malocclusion than male subjects. Our study finding of equality of males and females in attitude toward the orthodontic experience is not, however, consistent with previous studies that demonstrated gender differences in awareness and the knowledge of the orthodontic treatment of treated and untreated subjects. ${ }^{14,15}$ Our finding can be explained by the differences in traditions and social customs of the study subjects. Moreover, our obser-

Table 5: Bivariate correlation between items of orthodontist-patient communication, awareness, and knowledge of orthodontic treatment for male (57) and female (45) previously treated subjects

\begin{tabular}{llllll}
\hline & \multicolumn{2}{c}{ Males } & & \multicolumn{2}{c}{ Females } \\
\cline { 2 - 3 } \cline { 5 - 6 } & Pearson correlation & Significant (one-tailed) & & Pearson correlation & Significant (one-tailed) \\
\hline Communication vs awareness & 0.170 & 0.103 & 0.041 & 0.394 \\
Communication vs knowledge & $0.441^{*}$ & 0.001 & 0.030 & 0.423 \\
Communication vs total items & $0.401^{*}$ & 0.001 & 0.399
\end{tabular}

*Difference was statistically significant at the 0.01 level (one-tailed) 
vation may indicate that when both sexes are at identical educational levels, they are equally knowledgeable with respect to oral health issues. ${ }^{16}$

Orthodontic therapy in general is time consuming and implemented for several monthly appointments during which the orthodontist communicates with his patients. ${ }^{17}$ In our study, recently perceived treatment subjects were highly positive in general perception regarding contact and communication with their treating orthodontists. However, when comparing patient-orthodontist communications regarding the gender, the correlation was significantly higher in male treated subjects than females. These unexpected gender differences of relationship between communications and increased knowledge for the male patients could be explained by the traditions and social customs of the study subjects. Often the communication between native female patient and the treating orthodontist is so difficult in these cultures. Thus, increasing clinically oriented education for female patients using effective learning resources, such as educational videos and brochures about principles of orthodontic therapy may be helpful in the region.

The results of this study indicated that the association of orthodontist-patient communication, in general, was more significant with the knowledge of orthodontic treatment than with general awareness and that might be explained by the fact that communications had been focused on information related to the orthodontic technique. Previously, it has been demonstrated that subjects with orthodontic treatment were more aware and had better attitude toward orthodontic treatment than untreated subjects. ${ }^{18,19}$ In this study, the general attitudes of treated and untreated subjects toward orthodontics were evaluated by a self-reported questionnaire, thus the scales used might be less reliable. However, the uniform demographic and social criteria of the study sample, as well as internal consistency of the study, were quite satisfactory. Our study also showed that the orthodontic treated patients indicated significant positive perception in contacting and discussing the different issues related to the diagnosis of their malocclusion and treatment plan and that the perception had not been evaluated clinically by assessing occlusal outcomes and final result satisfaction. Therefore, this study may need to be confirmed by clinical assessment of occlusal and soft tissue outcomes and the extent of patient satisfaction.

\section{CONCLUSION}

The study can be concluded as:

- Subjects who recently received orthodontic treatment have more knowledge and awareness about orthodontic treatment than subjects who do not have orthodontic experience.
- Gender equality related to knowledge and awareness toward orthodontic treatment exists in orthodontic treated patients.

- Significant association between communication and knowledge of orthodontic treatment in male orthodontic patients exists as compared with female patients.

\section{ACKNOWLEDGMENTS}

Authors would like to thank the clinical director of Dentistry College, Jazan University, Dr Abdullah Meshni, Kingdom of Saudi Arabia, for his kind help to conduct this survey on the orthodontic patients of the college specialty clinics. Author also thank the assistants of the orthodontic clinics, Darlene AD and Karen Valdes, for their efforts in supervising the data collection. The authors appreciate the efforts of the employees of the College of Dentistry for facilitating and preparing the questionnaires.

\section{REFERENCES}

1. Lukez A, Pavlic A, Trinajstic Zrinski M, Spalj S. The unique contribution of elements of smile aesthetics to psychosocial well-being. J Oral Rehabil 2015 Apr;42(4):275-281.

2. Wright FA. Children's perception of vulnerability to illness and dental disease. Community Dent Oral Epidemiol 1982 Feb;10(1):29-32.

3. Bos A, Hoogstraten J, Prahl-Andersen B. Attitudes towards orthodontic treatment: a comparison of treated and untreated subjects. Eur J Orthod 2005 Apr;27(2):148-154.

4. Essamet M, Darout IA. Awareness and behavior related to orthodontic treatment among Jazan University students, Kingdom of Saudi Arabia. J Dent Oral Hyg 2016 Mar;8(3): 12-17.

5. Bertakis K. Recognition and empowerment: an effective approach to enlisting patient cooperation. J Fam Pract 1977;5: 217-222.

6. Fields RS. Influencing patient cooperation. J Clin Orthod 1980 Jun;14(6):417-421.

7. Kaplan AS, Rubin JG. A dental phobia clinic. N Y State Dent J 1984 Oct;50(8):491-492.

8. Knierim R. Use of stamped chart notations to enhance patient compliance. J Clin Orthod 1992 Jul;26(7):394-395.

9. Milgrom P, Vignehsa H, Weinstein P. Adolescent dental fear and control: prevalence and theoretical implications. Behav Res Ther 1992 Jul;30(4):367-373.

10. Sinclair P. The readers' corner. J Clin Orthod 1989;23(12): 795-796.

11. Tedesco, LA. Symposium overview: what did they say and what does it mean? In: McNamara, JA.; Trotman, C., editors. Creating the compliant patient. Ann Arbor: Center for Human Growth and Development, University of Michigan; 1997.

12. Ramsay DS. Patient compliance with oral hygiene regimens: a behavioural self-regulation analysis with implications for technology. Int Dent J 2000;(Suppl) Creating A Successful:304-311.

13. Spikmans FJ, Brug J, Doven MM, Kruizenga HM, Hofsteenge GH, van Bokhorst-van der Schueren MA. Why 
do diabetic patients not attend appointments with their dietitian? J Hum Nutr Diet 2003 Jun;16(3):151-158.

14. Lahti S, Tuutti H, Hausen H, Kääriäinen R. Opinions of different subgroups of dentists and patients about the ideal dentist and the ideal patient. Community Dent Oral Epidemiol 1995 Apr;23(2):89-94.

15. DiMatteo MR, McBride CA, Shugars DA, O’Neil EH. Public attitudes toward dentists: a U.S. household survey. J Am Dent Assoc 1995 Nov;126(11):1563-1570.

16. Darout IA, Mubarky AY, Abduh MA. Oral health related knowledge and behavior among secondary school students in Jazan region, Kingdom of Saudi Arabia. Am J Health Res 2016;4(5):138-142.

17. Bhatarai P, Shrestha RM. Comparative study of duration of orthodontic treatment among Nepalese adolescent and adult patients. Orthod J Nepal 2011;1(1):28-30.

18. Shrestha RM, Bhattarai P, Dhakal J, Shrestha S. Knowledge, attitude and practice of patients towards orthodontic treatment: a multi-centric study. Orthod J Nepal 2014 Oct;4(1):6-11.

19. Siddegowda R. An epidemiological survey on awareness towards orthodontic treatment in South Indian school children. Open J Dent Oral Med 2013 Sep;1(1):5-8. 